\title{
Implementação da Rede Cegonha em uma Regional de Saúde do estado de Goiás: o que os indicadores de saúde mostram sobre atenção materno-infantil?
}

\author{
Implementation of the Rede Cegonha (Stork Network) in a Regional Health \\ Department in the state of Goiás, Brazil: what do health indicators show \\ about maternal and child care?
}

\section{Implementación de la Rede Cegonha (Red Cigüeña) en una Dirección Regional de Salud del estado de Goiás, Brasil: ¿Qué muestran los indicadores de la salud sobre la atención materno-infantil?}

Thaís Rocha Assis ${ }^{1, a}$

rochafisio.thais@gmail.com | https://orcid.org/0000-0001-5908-2870

Virginia Oliveira Chagas ${ }^{2, a}$

virginiafarm@gmail.com | https://orcid.org/0000-0003-3470-7234

Raissa de Melo Goes ${ }^{3, b}$

raissa.mgoes@gmail.com | https://orcid.org/o000-0002-5281-4394

Nathany Souza Schafauser ${ }^{2, c}$

nathany-92@hotmail.com | https://orcid.org/oooo-0002-7790-7191

Klara Gomes Caitano ${ }^{2, d}$

klaragomes.fisio@gmail.com | https://orcid.org/0000-0002-4315-0514

Renatha Almeida Marquez ${ }^{2, e}$

renathamarquez@gmail.com | https://orcid.org/o0oo-0002-5460-3646

${ }^{1}$ Universidade Federal de Goiás, Regional Goiânia. Goiânia, GO, Brasil.

2 Universidade Federal de Goiás, Regional Jataí. Jataí, GO, Brasil.

${ }^{3}$ Instituto Gerar. São Paulo, SP, Brasil.

a Doutorado em Ciências da Saúde pela Universidade Federal de Goiás.

b Especialização em Psicologia Perinatal e Parental pelo Instituto Gerar.

'Especialização em Urgência e Emergência pela Secretaria de Saúde do Estado de Goiás.

d Graduação em Fisioterapia pela Universidade Federal de Goiás.

e Especialização em Terapia Manual pelo Instituto de Ensino Superior Blauro Cardoso de Mattos.

\section{Resumo}

Os indicadores de morbidade e de mortalidade materno-infantis são essenciais no contexto da organização da Rede Cegonha, no âmbito do Sistema Único de Saúde (SUS). Esta rede organiza-se para assegurar o acesso, o acolhimento e a resolutividade, por meio de um modelo de atenção voltado para o pré-natal, parto e nascimento, puerpério e sistema logístico. Este artigo apresenta um estudo ecológico desta rede em uma Regional de Saúde, realizado com uso de dados dos Sistemas de Informação de Saúde do Departamento de Informática do SUS. Verificou-se um percentual crescente de gestantes que realizaram sete ou mais consultas pré-natais. No entanto, poucas concluíram a assistência pré-natal, porque não realizaram a consulta de puerpério. Observou-se uma taxa de mortalidade infantil próxima à da meta estipulada pela ONU. Identificou-se um aumento das cesáreas e uma redução de partos normais, além de uma alta mortalidade materna. Esses indicadores apontam para a necessidade de melhoria da qualidade da atenção pré-natal e da assistência ao parto.

Palavras-chave: Serviços de saúde materno-infantil; Indicadores básicos de saúde; Mortalidade materna; Mortalidade infantil; Assistência à saúde; Saúde da mulher. 


\begin{abstract}
Indicators of maternal-child morbidity and mortality are essential in the context of the organization of the Rede Cegonha (Stork Network), within the scope of the Unified Health System (SUS - Sistema Único de Saúde). This network is organized to ensure access, protection and successful resolution, through a model of attention focused on prenatal, delivery and childbirth, puerperium (or postpartum period) and logistic system. This article presents an ecological study of this network in a Regional de Saúde (Regional Health Department) that was carried out using data from the SUS Department of Informatics of the Health Information Systems. An increasing percentage of pregnant women who had seven or more prenatal visits could be observed. However, few pregnant women completed prenatal care because they have not sought for the puerperium consultation. A child mortality rate close to the target set out in the United Nations Sustainable Development Goals was observed. An increase in caesarean sections and a reduction in normal deliveries and at the same time a high maternal mortality were identified. These indicators point to the need to improve the quality of prenatal and delivery care.
\end{abstract}

Keywords: Maternal-child health services; Health status indicators; Maternal mortality; Child mortality; Health care; Woman health.

\title{
Resumen
}

Los indicadores de morbilidad y de mortalidad materno-infantil son esenciales en el contexto de la organización de la Rede Cegonha (Red Cigüeña), en el ámbito del Sistema Único de Salud (SUS - Sistema Único de Saúde). Esa red se organiza para asegurar el acceso, el acogimiento y la capacidad resolutiva, por medio de un modelo de atención dirigido al prenatal, parto, nacimiento, puerperio y sistema logístico. Este artículo presenta un estudio ecológico de la red en una Regional de Saúde (Dirección Regional de Salud) realizado utilizando datos de los Sistemas de Información de Salud del Departamento de Informática del SUS. Se ha verificado un porcentaje creciente de gestantes que realizaron siete o más consultas de prenatal. Sin embargo, pocas gestantes concluyeron la asistencia prenatal, porque no realizaron la consulta de puerperio. Se observó una tasa de mortalidad infantil próxima a la meta estipulada por la ONU. Se identificó un aumento de las cesáreas y una reducción de partos normales, además de una alta mortalidad materna. Esos indicadores apuntan la necesidad de mejorar la calidad de la atención prenatal y de la asistencia al parto.

Palabras clave: Servicios de salud materno-infantil; Indicadores de salud; Mortalidad materna; Mortalidad infantil; Prestación de servicios de salud; Salud de la mujer.

INFORMAÇÕES DO ARTIGO

Contribuição dos autores:

Concepção e desenho do estudo: Thaís Rocha Assis.

Aquisição, análise ou interpretação dos dados: Thaís Rocha Assis, Virgínia Oliveira Chagas, Nathany Souza Schafauser, Klara Gomes Caitano e Renatha Almeida Marquez.

Redação do manuscrito: Thaís Rocha Assis, Virgínia Oliveira Chagas e Raissa Melo de Goes.

Revisão crítica do conteúdo intelectual: Thaís Rocha Assis, Virgínia Oliveira Chagas, Raissa Melo de Goes e Nathany Souza Schafauser.

Declaração de conflito de interesses: não há.

Fontes de financiamento: não houve.

Considerações éticas: o estudo foi realizado conforme as Diretrizes e Normas Regulamentadoras de Pesquisa Envolvendo Seres Humanos (Resolução 466/12 do Conselho Nacional de Saúde) e foi aprovado pelo Comitê de Ética em Pesquisa da Universidade Federal de Goiás (parecer número 965.374).

Agradecimentos/Contribuições adicionais: agradecemos a toda a equipe da iniciação científica da UFG de 2017 e à Secretaria Municipal de Saúde de Jataí-GO.

Histórico do artigo: submetido: 4 set. 2018 | aceito: 30 jul. 2019 | publicado: 20 dez. 2019.

Apresentação anterior: apresentado no $14^{\circ}$ Congresso de Pesquisa, Ensino e Extensão da Universidade Federal de Goiás no Seminário de Iniciação Científica.

Licença CC BY-NC atribuição não comercial. Com essa licença é permitido acessar, baixar (download), copiar, imprimir, compartilhar, reutilizar e distribuir os artigos, desde que para uso não comercial e com a citação da fonte, conferindo os devidos créditos de autoria e menção à Reciis. Nesses casos, nenhuma permissão é necessária por parte dos autores ou dos editores. 


\section{Introdução}

Os indicadores de morbidade e mortalidade materno-infantis são essenciais no contexto da organização e funcionamento da Rede Cegonha, no âmbito do Sistema Único de Saúde (SUS). Esta é uma rede temática instituída pelo Ministério da Saúde (MS), em 2011, por meio da Portaria ${ }^{0}{ }^{0}$ 1.459, de 24 de junho ${ }^{1}$ e alterada pela Portaria $\mathrm{n}^{0}$ 2.351, de 5 de outubro², para melhorar e reduzir estes indicadores que ainda são altos no Brasil, apesar de iniciativas anteriores para efetivar a rede de cuidados materno e infantil, como o Programa de Humanização no Pré-natal e Nascimento (PHPN)3.

A Rede Cegonha organiza-se para assegurar o acesso, o acolhimento e a resolutividade, por meio de um modelo de atenção voltado ao pré-natal, parto e nascimento, puerpério e sistema logístico, que inclui o transporte sanitário e a regulação ${ }^{1}$. Dessa forma, a rede possui quatro componentes: o componente pré-natal, que garante às mulheres o acesso ao pré-natal de qualidade, o acolhimento com avaliação e classificação de risco e vulnerabilidade, a vinculação da gestante à unidade de referência (Unidade Básica de Saúde e maternidade) e o transporte; o componente parto e nascimento, que garante a segurança na assistência; o componente puerpério e atenção integral à saúde da criança, com assistência a crianças de 0 a 24 meses de qualidade e com resolutividade, além do acesso às ações de planejamento reprodutivo; e o sistema logístico, transporte sanitário e regulação, que garante o acesso aos serviços citados anteriormente ${ }^{2}$.

A importância dos indicadores materno-infantis se dá porque permitem o conhecimento da situação de saúde local, funcionando, assim, como uma matriz diagnóstica que permite aumentar a efetividade das ações da Rede Cegonha em cada região, de acordo com os fatores de risco locais identificados ${ }^{4}$.

Dessa forma, a mensuração da mortalidade materna é considerada um indicador do nível de desenvolvimento da saúde e pode refletir a qualidade da atenção à saúde das mulheres ${ }^{5}$. A taxa de mortalidade neonatal reflete as condições de assistência à gravidez, ao parto e ao período perinatal ${ }^{6}$. O número de consultas pré-natais com captação precoce e a consulta de puerpério demonstram as condições de acesso e a cobertura dos serviços de saúde7.

No Brasil, a meta a ser cumprida como parte do quinto Objetivo de Desenvolvimento do Milênio é a redução de três quartos da razão de mortalidade materna até $2015^{8}$, e alguns fatores têm dificultado a melhoria desse indicador, como a fragmentação das ações e dos serviços de saúde; o financiamento insuficiente; as falhas na gestão dos serviços de saúde; e, ainda, a produção do cuidado centrada na realização de procedimentos sem evidências científicas, com “intervenções” desnecessárias na gestação, no parto e no nascimento7.

Nesse sentido, a Rede Cegonha tem sido implementada de forma gradativa no Brasil, em todo o território nacional, respeitando alguns critérios epidemiológicos como a taxa de mortalidade infantil, a razão de mortalidade materna e a densidade populacional, sendo responsabilidade dos estados e municípios elaborarem seus planos de ação para o repasse dos recursos financeiros, o monitoramento e a avaliação da implantação da Rede Cegonha7.

É sobre o processo de implementação da Rede Cegonha que este estudo pretende refletir e contribuir para o fortalecimento das ações de atenção materna e infantil e teve como objetivo apresentar os principais indicadores relacionados à implementação da Rede Cegonha na Regional de Saúde Sudoeste II, do estado de Goiás, entre os anos 2011 e 2015.

\section{Metodologia}

Trata-se de um estudo ecológico, com uso de dados secundários provenientes dos Sistemas de Informação de Saúde/Estatísticas Vitais do Departamento de Informática do Sistema Único de Saúde (DATASUS), Sistema de Informação de Nascidos Vivos (SINASC) ${ }^{9}$ e Sistema de Informação de Mortalidade (SIM) ${ }^{10}$, 
abertos para a consulta na internet, no Portal da Saúde do Ministério da Saúde. Também foram coletados dados no Sistema de Informações sobre o Pré-natal (SISPRENATAL), disponibilizado para acesso pela Secretaria Municipal de Saúde de Jataí, Goiás.

Foram incluídos na busca os dados relacionados aos nascidos vivos e à mortalidade, no período de 2011 a 2015, por residência da mãe e na Regional de Saúde Sudoeste II. Esta Regional está localizada no estado de Goiás, na região Centro-Oeste do Brasil, sendo jurisdicionados a ela dez municípios: Aporé, Caiapônia, Chapadão do Céu, Doverlândia, Jataí, Mineiros, Perolândia, Portelândia, Serranópolis e Santa Rita do Araguaia. Essa regional possui um total de $\mathbf{2 1 5 . 2 8 2}$ habitantes, sendo Jataí a sede ${ }^{11}$.

No SINASC, foram extraídos os seguintes dados: o número total de nascidos vivos e as taxas de cesárea e parto normal. No SIM, foram selecionadas as informações sobre óbitos infantis e óbitos de mulheres em idade fértil e óbitos maternos. Os óbitos infantis (em menores de um ano) foram categorizados em: mortalidade neonatal precoce (óbitos ocorridos de zero a seis dias de vida), mortalidade neonatal tardia (de sete a 27 dias de vida) e mortalidade pós-neonatal (de 28 dias a um ano de vida). As informações sobre a mortalidade materna foram categorizadas por faixa etária e pela causa ${ }^{12}$.

As causas de óbitos maternos são divididas em morte materna obstétrica direta: "É aquela que ocorre por complicações obstétricas durante gravidez, parto ou puerpério devido a intervenções, omissões, tratamento incorreto ou a uma cadeia de eventos resultantes de qualquer dessas causas" resultante de doenças que existiam antes da gestação ou que se desenvolveram durante esse período, não provocadas por causas obstétricas diretas, mas agravadas pelos efeitos fisiológicos da gravidez"12.

Os dados coletados do SISPRENATAL foram: número total de gestantes com captação precoce (até a $12^{\text {a }}$ semana gestacional), número de gestantes com sete ou mais consultas pré-natal e uma consulta de puerpério até 42 dias pós-parto, também para o período de 2011 a 2015.

Após a consulta às informações nos sistemas, foram criadas tabelas que, posteriormente, foram exportadas para planilhas do Microsoft Excel 2010. Nas planilhas, foram calculados os indicadores e montados os gráficos e figuras com a síntese dos resultados. Para os cálculos de proporção, foi utilizado o número total da variável que foi analisada dividido pelo número total de nascidos vivos no mesmo local e ano e multiplicado por cem. Para os cálculos das taxas, foi utilizado o número total da variável a ser analisada dividido pelo número total de nascidos vivos no mesmo local e ano multiplicado por mil para a mortalidade infantil e por cem mil para a mortalidade materna.

Os resultados foram apresentados de acordo com os indicadores-chave para a implementação da Rede Cegonha: percentual de gestantes que realizaram sete ou mais consultas pré-natais e a consulta de puerpério, número de nascidos vivos e as taxas dos tipos de parto (importantes indicadores de atenção à saúde) e as taxas de óbitos infantis e o número absoluto de óbitos maternos por faixa etária (importantes indicadores de mortalidade) ${ }^{2}$.

\section{Resultados}

Na Figura 1, observamos que, em 2015, houve o maior número de gestantes com captação precoce, ou seja, que iniciaram o pré-natal até a $12^{\mathrm{a}}$ semana de gestação (1.266 gestantes). No entanto, pouquíssimas (cinco gestantes) concluíram a assistência pré-natal, ou seja, realizaram sete ou mais consultas pré-natais e uma consulta de puerpério (até 42 dias de pós-parto). Ao longo dos anos analisados, houve uma diminuição importante do número de gestantes que concluíram a assistência pré-natal. 


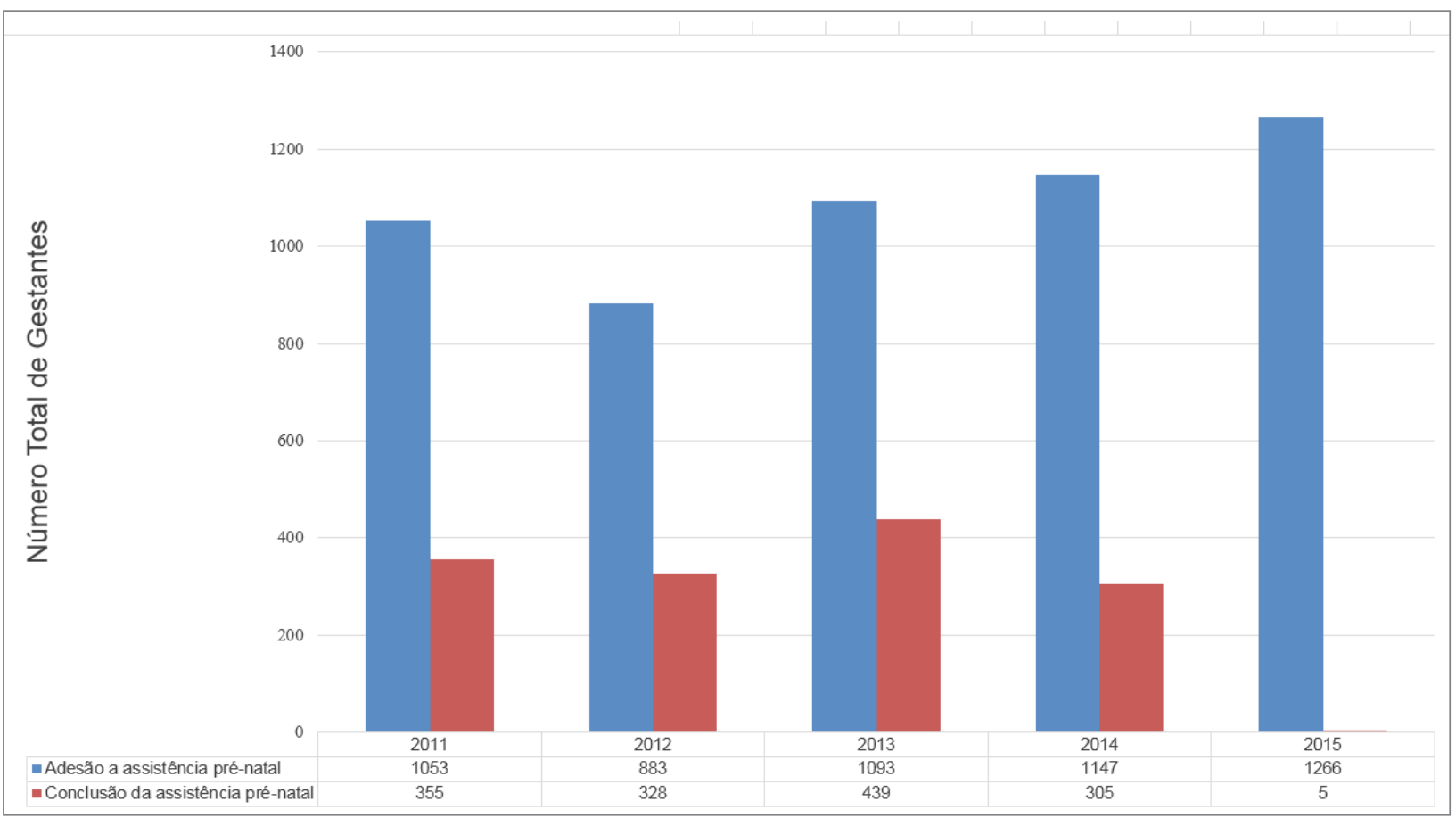

Figura 1 - Proporção das gestantes com captação precoce (em azul) e proporção de gestantes que realizaram sete ou mais consultas pré-natais e uma consulta de puerpério até 42 dias após o parto (em vermelho). Regional de Saúde Sudoeste II. 2017 Fonte: SISPRENATAL, 2017.

Na Figura 2, observamos que as taxas de cesáreas são superiores às de partos normais em todos os anos analisados. No ano de 2015, houve a maior taxa de cesarianas $(76,46 \%)$.

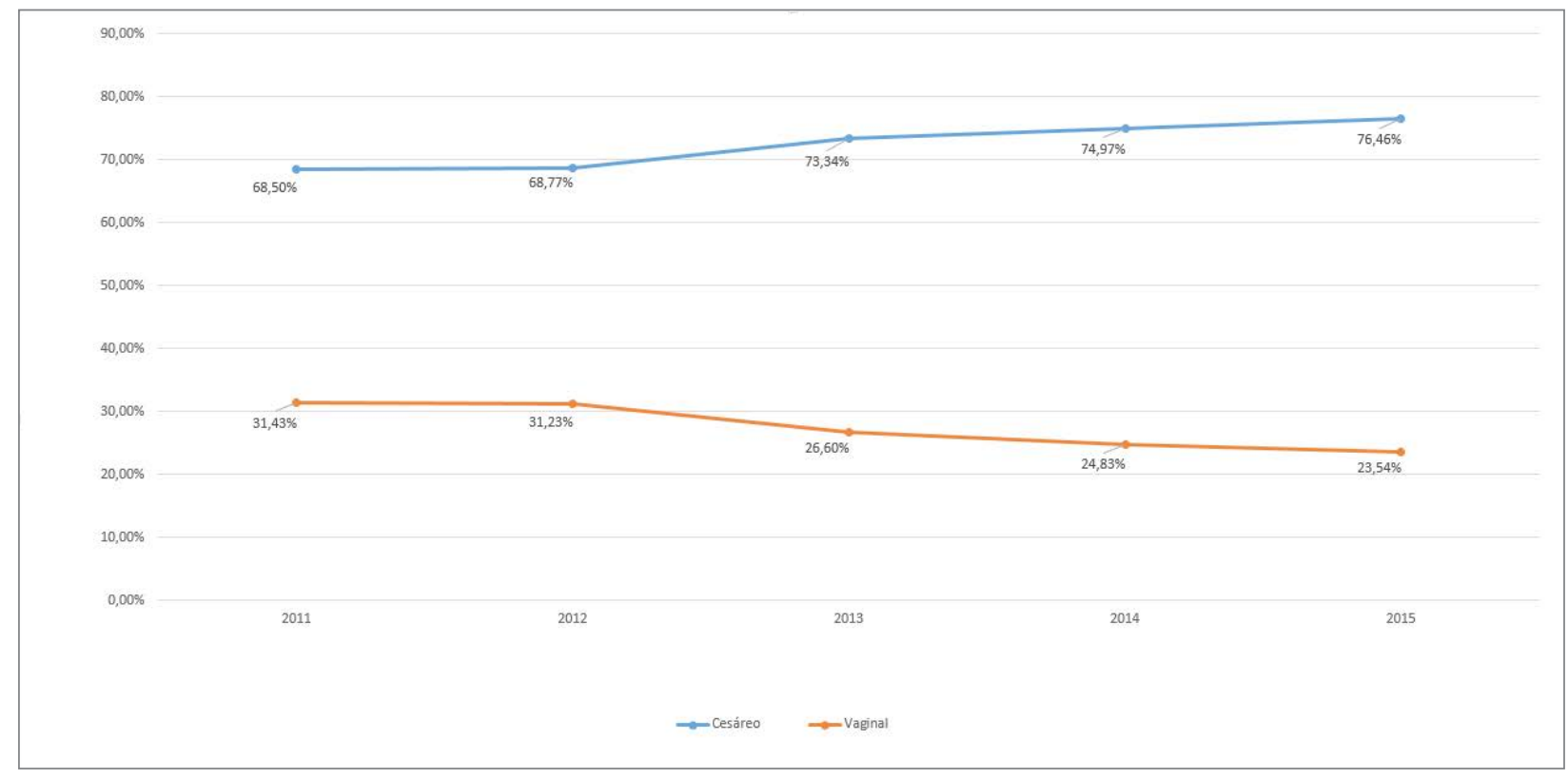

Figura 2 - Taxas de partos normais e cesáreas na Regional de Saúde Sudoeste II, nos anos de 2011 a 2015. Jataí, 2017 Fonte: SINASC, 2017.

Quanto à taxa de mortalidade infantil (recém-nascidos menores de um ano), a Figura 3 demonstra que a taxa de mortalidade neonatal precoce (o a 6 dias de vida) foi maior no ano de 2015 (10,63\%) e que a taxa de mortalidade pós-neonatal (28 a 365 dias) diminuiu ao longo dos anos analisados. Em números absolutos, houve 237 óbitos infantis no período analisado, tendo ocorrido 45 mortes em 2011, 48 em 2012, 50 em 2013, 41 em 2014 e 53 em 2015. 


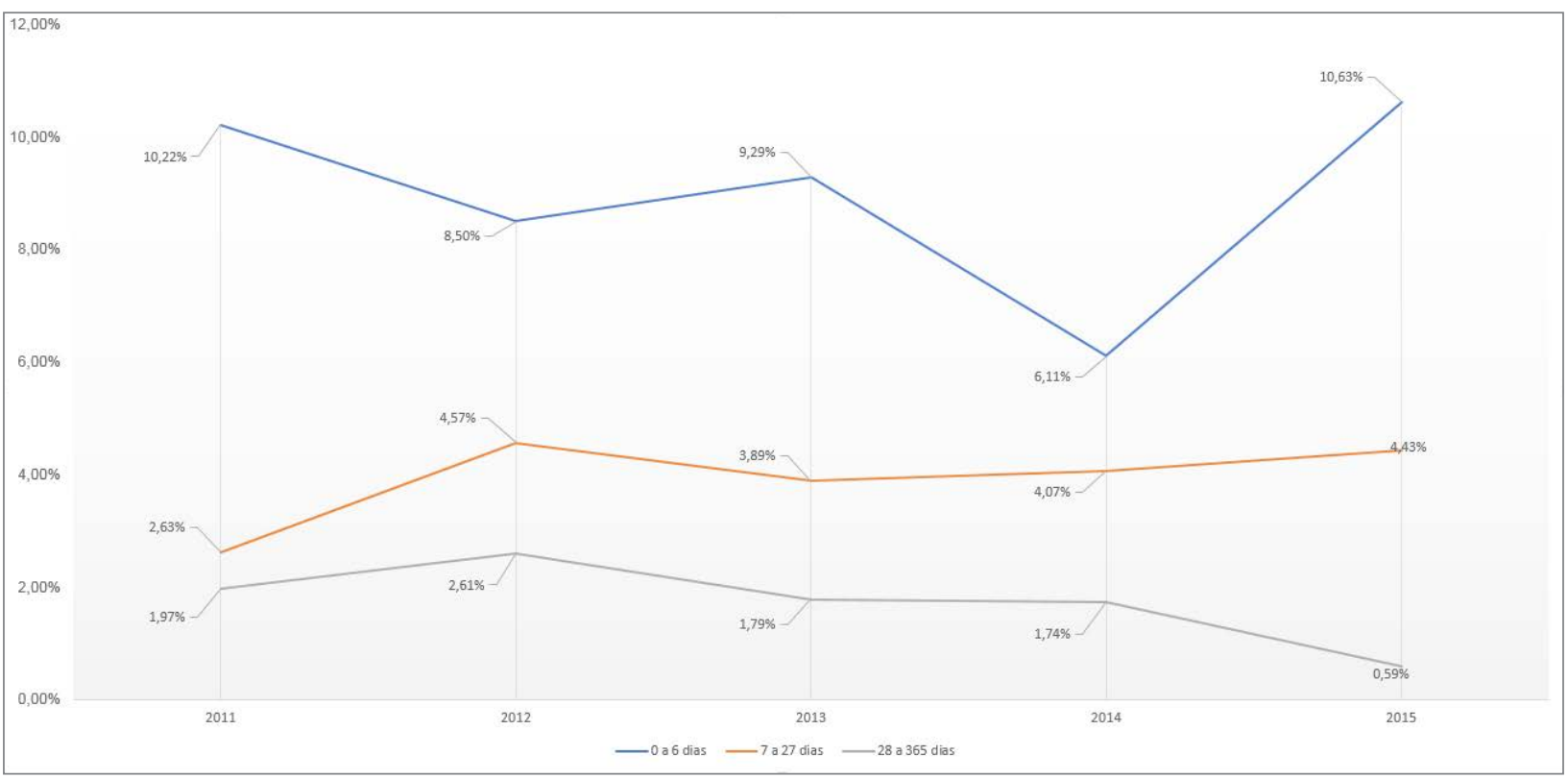

Figura 3 - Taxa de mortalidade infantil dividida nas suas categorias (neonatal precoce - 0 a 6 dias; tardia - 7 a 27 dias; e pós-neonatal - 28 a 365 dias), na Regional de Saúde Sudoeste II, nos anos de 2011 a 2015. Jataí, 2017 Fonte: SIM/SINASC, 2017.

Quanto à mortalidade materna, na Figura 4 observamos que, no ano de 2012, não houve registro de mortes maternas e que, no período de 2011 a 2015, houve um total de 13 mortes, cinco $(38,40 \%)$ delas de mães que estavam na faixa etária de 20 a 29 anos.

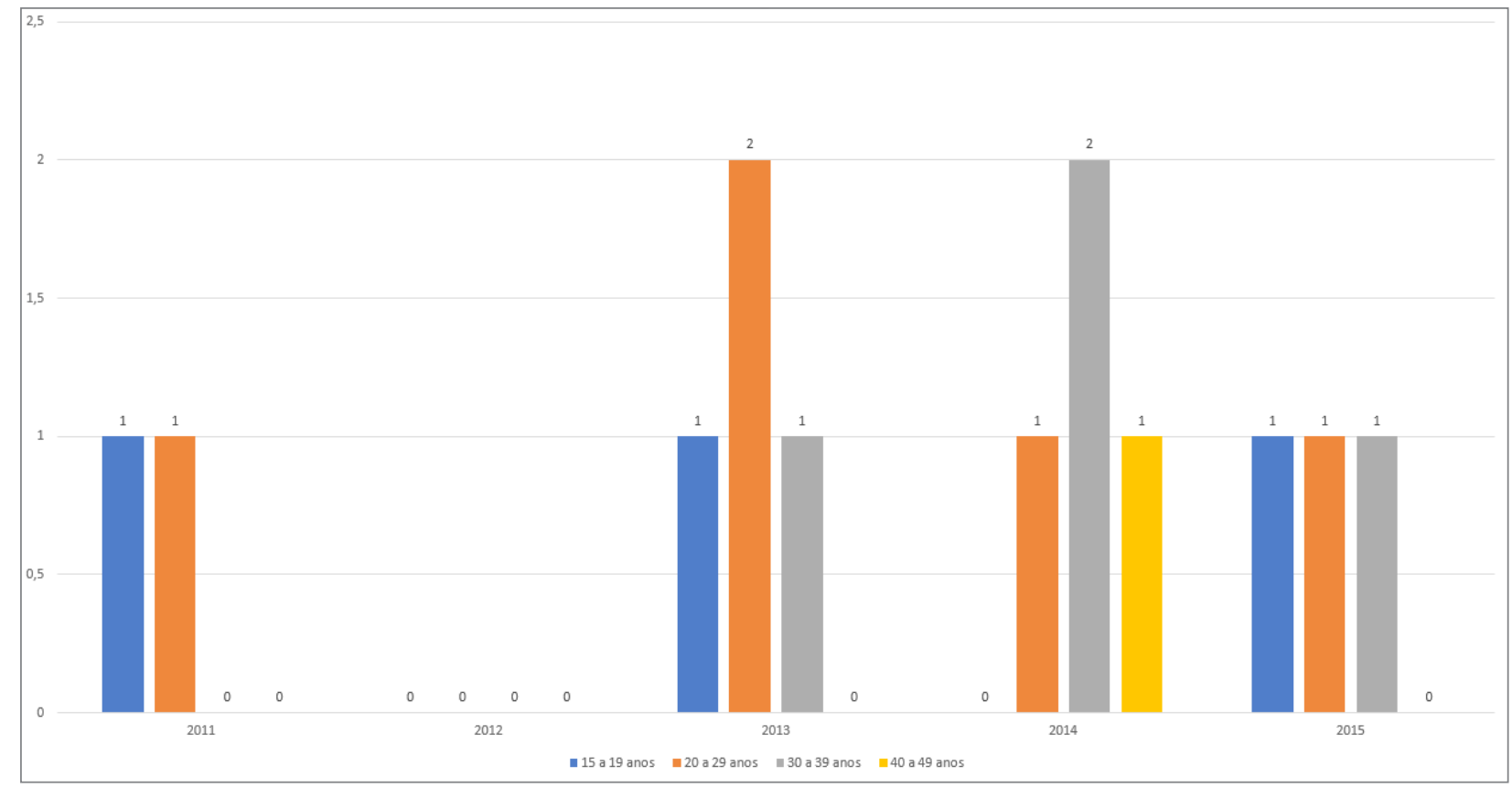

Figura 4 - Mortes maternas em número absoluto por faixa etária, na Regional de Saúde Sudoeste II, de 2011 a 2015. Jataí, 2017 Fonte: SIM, 2017.

Todas as causas dos óbitos maternos, nesse período, foram obstétricas diretas. De acordo com a categorização da Classificação Internacional de Doenças (CID10), os motivos dos óbitos, acompanhados aqui de seus códigos correspondentes, foram os seguintes: em 2011, um óbito por complicações venosas no puerpério (O87) e o outro por anormalidades da contração uterina (O62); em 2013, um óbito por eclâmpsia 
(O15), outro por hipertensão gestacional sem proteinúria significativa (O13), outro por retenção da placenta e membranas sem hemorragia (O73) e um óbito por outras infecções puerperais (O86); em 2014, um óbito por aborto espontâneo (Oo3), um óbito por hipertensão gestacional com proteinúria significativa (O14), outro óbito por eclâmpsia (O15) e outro por outras doenças maternas complicadas na gravidez, parto e puerpério (O99); em 2015, um óbito por hemorragia pós-parto (O72) e dois óbitos por outras doenças maternas complicadas na gravidez, parto e puerpério (O99).

Dessa maneira, cinco óbitos ocorreram durante a gravidez, parto ou aborto e oito durante o puerpério, até 42 dias. As desordens hipertensivas foram as principais causas dos óbitos, tendo ocorrido em quatro mulheres, o que corresponde a 30,7\% dos casos.

\section{Discussão}

Os indicadores de atenção à saúde apresentados neste estudo demonstraram um percentual crescente de gestantes que realizaram sete ou mais consultas pré-natais, e que houve um aumento de cerca de $5 \%$, de 2011 para 2015. No entanto, poucas gestantes - 355 em 2011 e apenas cinco em 2015 - concluíram a assistência pré-natal com a realização da consulta de puerpério (até 42 dias de pós-parto).

Acreditamos que a baixa adesão à consulta de puerpério tem relação com dificuldades de comunicação entre as gestantes e os profissionais de saúde durante o pré-natal na atenção básica, devido a consultas tecnicistas, que não permitem a criação de um espaço de acolhimento das angústias, receios e necessidades das gestantes ${ }^{13}$.

A Rede Cegonha prevê, no componente puerpério e atenção integral à saúde da criança, o acolhimento e o acompanhamento da puérpera e da criança na atenção básica com visita domiciliar na primeira semana após a realização do parto e nascimento e consulta puerperal entre o $30^{\circ}$ e o $42^{\circ}$ dia pós-parto ${ }^{1}$. Capacitar os profissionais da atenção básica para esse acolhimento e para a realização da visita domiciliar pode ser um caminho para melhorar a adesão das mulheres a essa assistência e para qualificar essa última.

Observamos, na Regional de Saúde analisada, altas taxas de cesáreas em todos os anos analisados. Verificamos, ainda, um maior número de mortes maternas entre 20 e 29 anos e que todas essas mortes foram por causa obstétrica direta. No ano de 2015, ocorreram três óbitos maternos para 3.385 nascidos vivos, o que corresponde, proporcionalmente, a um valor quase três vezes acima do pactuado com o Ministério da Saúde para 2015, que foi a taxa de 35 óbitos para cada cem mil nascidos vivos ${ }^{14}$.

As altas taxas de cesáreas e de mortalidade materna, apresentadas nesta pesquisa, apontam para o comprometimento da qualidade da atenção pré-natal e da assistência ao parto. Apesar do aumento do número de mulheres que realizaram sete ou mais consultas pré-natais, esse aumento da cobertura, que também é observado nacionalmente ${ }^{15}$, não resultou em qualidade dos serviços prestados.

Os altos índices de cesáreas na Regional de Saúde Sudoeste II não diferem da realidade apresentada em todo o Brasil, que possui as taxas mais altas de cesarianas do mundo. A região Centro-Oeste do Brasil já foi apontada, em outro estudo, como a região com maior índice de cesáreas ${ }^{16}$.

Algumas evidências apontam uma associação negativa entre a cesárea e a saúde no puerpério, mostrando que mulheres que tiveram seus filhos por meio da cirurgia cesariana apresentaram maior risco de depressão pós-parto e dificuldades com a amamentação ${ }^{17}$. Deste modo, é importante observar que as altas taxas de cesarianas juntamente com o baixo número de mulheres que voltam para as consultas de puerpério podem ser indicadores de que a saúde da mulher nesse período ainda precisa receber mais atenção por parte dos profissionais de saúde.

A assistência ao pré-natal ocorre na atenção básica, nível de atenção considerado porta de entrada do usuário do SUS e ordenador dos cuidados na rede de atenção à saúde ${ }^{18}$. Nesse nível de atenção, a prática assistencial é organizada por meio da Estratégia Saúde da Família (ESF) e é norteada por diretrizes como 
o cuidado centrado na pessoa, a longitudinalidade do cuidado, a resolutividade, a integralidade da assistência, o vínculo e a humanização. Nesse sentido, as mulheres deveriam ser informadas sobre as boas práticas e cuidados obstétricos adequados, sobre os benefícios do parto vaginal e serem preparadas para conduzir e ter autonomia sobre seu parto. No entanto, isso pouco acontece, resultando na opção das mulheres por cesárea eletiva ${ }^{16,19}$.

O comprometimento da qualidade da assistência ao parto, na regional de saúde analisada, pode ser verificado pelo fato de a maioria das mortes maternas serem por causas obstétricas diretas, com excesso de intervenções e tratamentos inadequados durante a gravidez, parto ou puerpério. Pesquisa recentemente realizada em uma maternidade pública no município sede da Regional de Saúde Sudoeste II, mostrou vários relatos de mulheres entrevistadas no pós-parto que evidenciaram uma assistência intervencionista ao parto, baseada em costumes, e sem evidências científicas, caracterizando situações de violência obstétrica ${ }^{20}$. É importante ressaltar que o momento do trabalho de parto é de uma sensibilidade única e os acontecimentos e interações que ocorrem durante este período têm consequências psicológicas intensas e duradouras ${ }^{21}$, como por exemplo uma quebra do vínculo e confiança na equipe de saúde, justificando o não retorno das puérperas para as consultas de pós-parto.

Dessa forma, a melhoria da assistência ao parto não depende de novos conhecimentos, mas da garantia da acessibilidade e da utilização mais efetiva do conhecimento científico e tecnológico existente ${ }^{6}$.

Ainda acreditamos ser importante enfatizar a relação entre as altas taxas de cesárea ao longo dos anos analisados e o aumento das mortes maternas, na pesquisa aqui apresentada. Os resultados obtidos em uma revisão sistemática com metanálise indicaram que mulheres de parto cesárea têm maior chance de morte materna e infecção pós-parto ${ }^{22}$. Além da cesárea estar relacionada a taxas de mortalidade materna aproximadamente quatro a cinco vezes maiores que o parto vaginal, encontra-se associada ao aumento da morbidade e mortalidade perinatal ${ }^{23}$. Isto também pode ser observado, neste estudo, para o ano de 2015, quando houve a maior taxa de óbitos neonatais precoces e a maior taxa de cesarianas. Outro estudo aponta que as altas taxas de cesarianas levam também a uma maior taxa de admissão em UTI neonatal e a um maior uso de ventilação mecânica em recém-nascidos ${ }^{24}$.

Sabe-se que, atualmente, no Brasil, o principal componente da mortalidade infantil é a mortalidade neonatal precoce, o que reflete as condições de assistência à gravidez, ao parto e ao período perinatal ${ }^{6,25}$. Nos primeiros dias de vida, o neonato encontra-se no período de adaptações ao meio extrauterino e, deste modo, está suscetível a óbito por múltiplos fatores que procedem de determinantes socioeconômicos, biológicos e associados à atenção à saúde no pré-natal, parto e puerpério ${ }^{6}$. Para a redução da mortalidade infantil nesses casos, deve-se melhorar a estrutura assistencial médica hospitalar, instalar uma efetiva rede de maternidades, berçários e Unidades de Tratamento Intensivos pediátricas e neonatais ${ }^{6}$.

A taxa de mortalidade infantil no Brasil vem diminuindo ao longo dos anos. Em 2015, ela foi de 13,82 crianças mortas para cada mil nascidas vivas, superando a meta estabelecida pela Organização das Nações Unidas (ONU) que foi de 15 mortes infantis para cada mil nascidos vivos ${ }^{26}$. A Regional de Saúde analisada, nesta pesquisa, teve uma taxa acima da nacional, no ano de 2015, pois houve 15,65 óbitos infantis para cada mil nascidos vivos, porém muito próxima à meta estipulada pela ONU.

Embora a taxa de mortalidade infantil na regional em questão esteja maior que a nacional, ela apresentou melhores resultados do que a mortalidade materna. Assim, é possível notar como a saúde do recém-nascido tem sido priorizada em relação aos cuidados com a saúde da mulher. Há uma aparente invisibilidade da mulher no período pós-parto e a assistência de saúde parece não reconhecer este período como parte do ciclo gravidez e parto, negligenciando a importância da continuidade do cuidado. 
Ao entrar no puerpério, a mulher está bastante fragilizada, pois percebe que precisa dar conta de uma vida para além da sua própria e dada a romantização cultural da maternidade, que é tida como o momento em que a mulher sente-se plena, muitas vezes, a puérpera não se sente autorizada a reclamar dessa nova vida, muito menos pedir ajuda. A mulher sente que precisa dar conta de tudo para ser considerada uma boa mãe, negligenciando muitas vezes o autocuidado em prol do cuidado do recémnascido. É por isso que é importante ela ter acesso a uma rede onde se sinta acolhida e que esteja atenta aos sinais físicos e psíquicos sem naturalizá-los ou patologizá-los.

A efetividade e a sustentabilidade das ações da Rede Cegonha só poderão ter sucesso a partir da estruturação de um modelo organizacional que garanta a continuidade dos cuidados com a saúde materna e infantil para a população do território, e com a responsabilização pelos resultados sanitários referentes a essa população ${ }^{27}$. Implementar um Conselho Gestor na maternidade e outros dispositivos de cogestão são importantes iniciativas para auxiliar a implementação da rede ${ }^{1}$.

Enfatizamos a necessidade do fortalecimento da atenção básica, uma vez que é a ordenadora e coordenadora da rede de atenção à saúde, através da Estratégia Saúde da Família (ESF) e do Núcleo Ampliado de Saúde da Família e Atenção Básica (NASF-AB), para garantir a resolutividade, longitudinalidade, integralidade do cuidado e humanização da assistência à saúde materna e infantil.

Espera-se que este estudo possa fornecer alguns subsídios para a gestão do SUS repensar as políticas públicas voltadas à saúde das mulheres e fortalecer o respeito e a confiança essenciais à corresponsabilidade dos envolvidos no cuidado puerperal.

\section{Conclusão}

Os indicadores de atenção à saúde apresentados demonstraram um percentual crescente de gestantes que realizaram sete ou mais consultas pré-natais. No entanto, poucas gestantes concluíram a assistência pré-natal, porque não realizaram a consulta de puerpério. Observou-se um número crescente de nascidos vivos na Regional de Saúde analisada. Quanto ao tipo de parto, observaram-se altas taxas de cesáreas.

Quanto aos óbitos maternos por faixa etária, verificou-se um maior número de mortes maternas entre 20 e 29 anos. Todas as mortes maternas foram por causa obstétrica direta, e a sua maioria ocorreu durante o puerpério. Quanto à mortalidade infantil, observou-se que a taxa de mortalidade neonatal precoce (óbitos ocorridos entre o e 6 dias de vida) apresentou o maior aumento quando comparada às mortalidades tardia e pós-natal.

As altas taxas de cesáreas e a alta mortalidade materna, apresentadas na pesquisa que fundamenta este artigo, apontam para a necessidade de melhorar a qualidade da atenção pré-natal e da assistência ao parto. Ações como a qualificação dos profissionais de saúde que atuam na maternidade podem contribuir para reduzir esses indicadores, uma vez que aumentariam as boas práticas de atenção ao parto e ao nascimento, baseadas em evidências científicas, o que diminuiria as intervenções desnecessárias como, por exemplo, as cesáreas eletivas.

O fortalecimento da assistência ao pré-natal na atenção básica, por meio da qualificação dos profissionais da ESF e do NASF-AB, com consultas mais humanizadas, interprofissionais e menos tecnicistas, podem garantir o vínculo das mulheres à UBS e aumentar as chances de voltarem para a consulta de puerpério. Além disso, é fundamental enfatizar a importância da visita domiciliar, na primeira semana após o parto, para a identificação das complicações que podem levar ao óbito materno e infantil no pós-parto e, assim, permitir a intervenção mais rápida dos profissionais de saúde. Capacitar os agentes comunitários de saúde para identificarem essas complicações, é fundamental para garantir a efetividade dessa visita. 


\section{Referências}

1. Ministério da Saúde (BR). Portaria no 1.459 de 24 de junho de 2011. Institui, no âmbito do Sistema Único de Saúde (SUS), a Rede Cegonha. DOU. 2011 jun. 25.

2. Ministério da Saúde (BR). Portaria no 2.351/GM/MS de 5 de outubro de 2011. Altera a Portaria no 1.459/ GM/MS, de 24 de junho de 2011, que institui, no âmbito do Sistema Único de Saúde (SUS), a Rede Cegonha. DOU. 2011 jul. 27.

3. Ministério da Saúde (BR). Portaria $n^{\circ} 569,01$ de junho de 2000. Institui, no âmbito do Sistema Único de Saúde (SUS), o Programa de Humanização no Pré-Natal e Nascimento. DOU. 2000 jun. 01.

4. Silva CMCD, Gomes KRO, Rocha OAMS, Almeida IMLM, Moita NJM. Validade, confiabilidade e evitabilidade da causa básica dos óbitos neonatais ocorridos em unidade de cuidados intensivos da Rede Norte-Nordeste de Saúde Perinatal. Cad Saúde Pública [Internet]. 2013 mar. [citado em 2018 ago. 29];29(3):547-56. doi: http://dx.doi.org/10.1590/S0102-311X2013000300012.

5. Ferraz L, Bordignon M. Mortalidade materna no Brasil: uma realidade que precisa melhorar. Rev Baiana Saúde Pública [Internet]. 2012 [citado em 29 ago. 2018];36(2):527-38. Disponível em: http://files.bvs. br/upload/S/0100-0233/2012/v36n2/a3253.pdf.

6. Nascimento RM, Leite AJM, Almeida NMGS, Almeida PC, Silva CF. Determinantes da mortalidade neonatal: estudo caso-controle em Fortaleza, Ceará, Brasil. Cad Saúde Pública [Internet]. 2012 mar. [citado em 2018 ago. 29];28(3):559-72. doi: http://dx.doi.org/10.1590/S0102-311X2012000300016.

7. Ministério da Saúde (BR). Secretaria de Atenção à Saúde. Manual prático para implementação da Rede Cegonha. Brasília: Ministério; 2011.

8. United Nations. General Assembly: fifty-fifth session: Resolution adopted by the General Assembly: United Nations Millennium Declaration [without reference to a Main Committee (A/55/L.2)]. New York: The United Nations; 2000.

9. Datasus: tecnologia da informação a serviço do SUS. Nascidos vivos Brasil 2017 [Internet]. Brasília: Ministério da Saúde [citado em 2017 jul. 11]; 2017. Disponível em: https://bit.ly/34lfDrh.

10. 10 Ministério da Saúde (BR). Portal da Saúde. Informações de saúde [Tabnet]. Brasília: O Ministério; 2017 [citado em 2017 jul. 11]; Disponível em: http://www2.datasus.gov.br/DATASUS/index. php?area $=0205 \&$ id $=6937$.

11. Vilela AR. Regional de Saúde Sudoeste II: Jataí [Internet]. 2016 [citado em 2016 abr. 19]. Disponível em: http://www.saude.go.gov.br/view/2418/regional-de-saude-sudoeste-ii-ndash-jatai.

12. Ministério da Saúde (BR). Secretaria de Atenção à Saúde. Departamento de Ações Programáticas Estratégicas. Manual dos Comitês de Mortalidade Materna. 3. ed. Brasília: O Ministério; 2009. (Série A. Normas e Manuais Técnicos).

13. Corrêa MSM, Feliciano KVO, Pedrosa EN, Souza AI. Acolhimento no cuidado à saúde da mulher no puerpério. Cad Saúde Pública [Internet]. 2017 [citado em 2018 ago. 29];33(3):e00136215. doi: http:// dx.doi.org/10.1590/0102-311x00136215.

14. Ministério da Saúde (BR). Secretaria de Vigilância em Saúde. Nota técnica: mortalidade materna no Brasil. Objetivos de desenvolvimento do milênio: relatório nacional de acompanhamento. Brasília; o Ministério; 2010.

15. Viellas EF, Domingues RMSM, Dias MAB, Gama SGN, Theme FMM, Costa JV et al. Assistência pré-natal no Brasil. Cad Saúde Pública [Internet]. 2014[citado em 2018 ago. 29];30(Suppl 1):S85-S100. doi: http://dx.doi.org/10.1590/0102-311X00126013.

16. Leal MC, Pereira APE, Domingues RMSM, Filha MMT, Dias MAB, Nakamura-Pereira M et al. Intervenções obstétricas durante o trabalho de parto e parto em mulheres brasileiras de risco habitual. Cad. Saúde Pública [Internet]. 2014 [citado em 2018 ago. 29]; 30(Suppl 1): S17-S32. doi: http://dx.doi. org/10.1590/0102-311X00151513.

17. Salgado HO. A experiência da cesárea indesejada: perspectiva das mulheres sobre decisões e suas implicações no parto e nascimento [dissertação]. São Paulo: Universidade de São Paulo; 2013.

18. Starfield B. Atenção primária: equilíbrio entre necessidades de saúde, serviços e tecnologia [Internet]. Brasília: Unesco, Ministério da Saúde; 2002 [citado em 2018 ago. 17]. Disponível em: https://www. nescon.medicina.ufmg.br/biblioteca/imagem/0253.pdf. 
19. Amorim MMR, Souza ASR, Port AMF. Indicações de cesariana baseadas em evidências: parte I. Femina [Internet]. 2010 [citado em 2018 ago. 28];38(8):415-22. Disponível em: https://bit.ly/36oEh]n.

20. Messias ALS, Chagas VO, Rezende FR, Prado GRP, Assis TR. Violência obstétrica: experiências de puérperas primíparas em uma maternidade pública. Cienc Cuid Saúde [Internet]. 2018 [citado em 2018 ago. 29];17(1):1-7. doi: http://dx.doi.org/10.4025/cienccuidsaude.v17i1.40258.

21. Leão MRC, Bastos MAR. Doulas apoiando mulheres durante o trabalho de parto: experiência do Hospital Sofia Feldman. Rev Latino-Am Enfermagem [Internet]. 2001 maio [citado em 2018 ago. 29];9(3):90-4. doi: http://dx.doi.org/10.1590/S0104-11692001000300014.

22. Mascarello KC, Horta BL, Silveira MFC. Complicações maternas e cesárea sem indicação: revisão sistemática e metanálise. Rev Saúde Pública [Internet]. 2017 [citado em 2018 ago. 28];51: 105. doi: http://dx.doi.org/10.11606/s1518-8787.2017051000389.

23. Villar J, Carroli G, Zavaleta N, Donner A, Wojdyla D, Faundes A et al. Maternal and neonatal individual risks and benefits associated with caesarean delivery: multicentre prospective study. BM] [Internet]; 2007[citado em 2018 ago. 28];335:1025. doi: https://doi.org/10.1136/bmj.39363.706956.55.

24. Salgado HO. Cuidado materno livre de danos e prevalência de depressão pós-parto: inquérito "Nascer no Brasil", Região Sudeste, 2011 e 2012 [tese]. São Paulo: Universidade de São Paulo; 2017.

25. França $\mathrm{E}$, Lansky S. Mortalidade infantil neonatal no Brasil: situação, tendências e perspectivas. In: Rede Interagencial de Informações para Saúde, organizador. Demografia e saúde: contribuição para análise de situação e tendências. Brasília: Organização Pan-Americana da Saúde; 2009. p. 83-112. (Série G. Estatística e Informação em Saúde; Série Informe de Situação e Tendências).

26. Portal Federativo; Secretaria de Governo. Notícias. Meta da ONU de reduzir a mortalidade infantil é superada em níveis nacional e municipais [Internet]. Brasília: O Portal; 2016 [citado em 2018 ago. 25]. Disponível em: https://bit.ly/2PEY8OC.

27. Marques CPC. Redes de atenção à saúde: a Rede Cegonha [Internet]. São Luís: Universidade Federal do Maranhão; Universidade Aberta do SUS; 2016 [citado em 2018 ago. 17]. Disponível em: https://ares. unasus.gov.br/acervo/handle/ARES/7564. 